\title{
Connectivity between visual and auditory cortices mediates the influence of argument strength on the effectiveness of smoking-cessation videos among smokers low in sensation seeking
}

This article was published in the following Dove Press journal:

Psychology Research and Behavior Management

\author{
Zhenhao Shi' \\ An-Li Wang ${ }^{2}$ \\ Catherine A Aronowitz ${ }^{3}$ \\ Joseph N Cappella ${ }^{4}$ \\ Daniel Romer ${ }^{3}$ \\ Daniel D Langleben ${ }^{1,3,5}$ \\ 'Center for Studies of Addiction, \\ Department of Psychiatry, University of \\ Pennsylvania Perelman School of \\ Medicine, Philadelphia, PA 19104, USA; \\ ${ }^{2}$ Department of Psychiatry, Icahn School \\ of Medicine at Mount Sinai, New York, \\ NY, 10029, USA; ${ }^{3}$ Annenberg Public \\ Policy Center, University of Pennsylvania, \\ Philadelphia, PA 19104, USA; ${ }^{4}$ Annenberg \\ School for Communication, University of \\ Pennsylvania, Philadelphia, PA 19104, \\ USA; ${ }^{5}$ Department of Behavioral Health, \\ Corporal Michael J. Crescenz Veterans \\ Administration Medical Center, \\ Philadelphia, PA, 19104, USA
}

Correspondence: Daniel D Langleben University of Pennsylvania Perelman School of Medicine, 3535 Market Street, Suite 500, Philadelphia, PA 19104, USA $\mathrm{Tel}+12157460107$

Email langlebe@pennmedicine.upenn.edu
Purpose: Argument strength (AS) is a validated measure of persuasiveness that has been identified as one of the key variables determining the effectiveness of video ads. Smokingcessation videos with high AS are more effective at reducing smoking behavior than videos with low AS. The neural processes that mediate the effects of AS on subsequent smoking have not been identified. In the present study, we tested whether the efficacy of high-AS smoking-cessation videos is determined by the level of integration of visual and auditory (ie, multisensory) processes. In addition, we tested differences in sensation seeking, which is repeatedly associated with smokers' sensitivity to cessation interventions.

Patients and methods: Using functional magnetic resonance imaging (fMRI), we recorded the brain response of 66 smokers randomly assigned to view either 16 high-AS or 16 low-AS smoking-cessation videos. Multisensory processing was assessed by the functional connectivity between brain regions that encoded visual and auditory information in the videos. Smoking behavior was indexed by the urine level of cotinine, a nicotine metabolite, immediately before and approximately 30 days after the fMRI session.

Results: We found a significant moderated mediation effect, such that the connectivity between visual and auditory cortices mediated the effect of AS on subsequent smoking, but only for smokers lower in sensation seeking. The prediction performance of the model was confirmed by leave-one-out cross-validation.

Conclusion: Our study suggests that audiovisual integration underlies the greater efficacy of high- vs low-AS smoking-cessation videos for individuals lower in sensation seeking. Highsensation-seeking smokers may be responsive to other characteristics of smoking-cessation videos. Keywords: smoking, health communication, functional magnetic resonance imaging, brain connectivity, sensation seeking

\section{Introduction}

Video ads or public service announcements have long been a key component of public health campaigns promoting smoking cessation ${ }^{1}$ and other health behaviors. ${ }^{2,3}$ Argument strength (AS) is a validated measure of persuasiveness that has been identified as one of the key variables determining video ads' effectiveness. ${ }^{4-6}$ Studies show that video messages with higher AS are more effective in changing attitudes and behaviors ${ }^{6-8}$ and that AS has distinct neural correlates. ${ }^{6,9}$ However, less is known about the brain mechanisms that mediate the effects of AS on behavioral outcomes. 
Neuroimaging studies suggest that brain response to health messages is a useful intermediate outcome measure of the effectiveness of these messages. ${ }^{6-9-13}$ In addition, unlike the purely visual pictorial and textual messages, videos carry both visual and auditory content that requires sustained externally paced processing over time. Therefore, effective video messaging depends on coherent integration of visual and auditory information, ${ }^{14}$ which is likely to engage multiple brain regions. ${ }^{15-18}$ However, prior analyses have not considered this multisensory nature of videos; by focusing on a single index of sensory impact (ie, "message sensation value"), ${ }^{12,13}$ these analyses were limited to regional neural responses without examining inter-regional connectivity. ${ }^{6,10-12}$ This perhaps explains why previously identified neural predictors of message effectiveness (such as medial prefrontal activity) did not mediate the greater effectiveness of high-AS videos. ${ }^{6}$

Health communication research has provided useful tools that use content analyses to capture the multisensory nature of health promotion videos. ${ }^{6,12,13,19-21}$ One is "message sensation value", a measure of sensor input that reflects the overall amount of visual and auditory features in a video. ${ }^{6,12,13,19}$ A more recent "I-squared" $\left(\mathrm{I}^{2}\right)$ measure quantitatively assesses the visual and auditory information introduced in each video. ${ }^{20,21}$ The $\mathrm{I}^{2}$ instrument contains an independent scale for visual and auditory modalities, which offers an advantage for the study of multisensory integration. Connectivity analysis is a rapidly growing area of neuroimaging research that investigates the functional interactions between brain regions. ${ }^{22}$ By examining the functional connectivity between brain regions that encode the two information modalities (ie, visual and auditory), one could obtain novel information about the multisensory encoding of videos. ${ }^{17,23,24}$ This may unravel the neurocognitive basis of the greater effectiveness of video messages that are higher in AS. The present study examined the connectivity between visual and auditory cortices as an index of multisensory processing in smokers watching either high- or low-AS videos. Our working hypothesis was that such connectivity while viewing smoking-cessation videos would mediate the effect of AS and subsequent change in smoking behavior.

Audience characteristics are another important factor that contributes to the outcomes of smoking-cessation messaging that have not been taken into account in previous research of neural correlates of AS. ${ }^{6}$ Studies indicate that sensation seeking may be a key characteristic modulating individuals' ability to change their behavior in response to health messages. Individuals that are high in sensation seeking tend to be more impulsive in the pursuit of rewarding experiences than low sensation seekers, ${ }^{25-28}$ resulting in greater difficulty in resisting a rewarding behavior, such as smoking. ${ }^{29-31}$ Therefore, it is possible that even when health messages are processed equally at the sensory level by high and low sensation seekers, those lower in sensation seeking benefit more readily from the high-AS video messages.

Using functional magnetic resonance imaging (fMRI), our previous study showed that smokers' neural and behavioral responses to AS are modulated by the sensory impact (ie, "Message Sensation Value") of smoking-cessation videos. ${ }^{6}$ In the present study, we conducted further analysis of these data by accounting for multisensory processing and sensation seeking. We aimed at identifying the neurocognitive mechanism that underlies the greater effectiveness of high-AS vs low-AS smoking-cessation video messages, and their potential interaction with sensation seeking. We examined the connectivity between the cortices that process visual and auditory information as a measure of multisensory integration. Smoking was assessed by the level of cotinine, a nicotine metabolite, in the urine, which is considered a reliable marker of actual smoking behavior. ${ }^{6,32-35}$ We hypothesized that 1) smoking-cessation videos with higher AS would produce greater brain connectivity between visual and auditory cortices, and that 2) greater connectivity would predict less smoking among the low-sensation-seeking smokers. We sought to test these hypotheses by examining the moderated mediation model where AS and future smoking behavior were the independent and outcome variables, respectively, and the connectivity between the visual and auditory cortices and sensation seeking were the mediator and moderator, respectively. ${ }^{36-39}$ Finally, we explored the predictive power of the model by testing how well subsequent smoking behavior was predicted by AS, sensation seeking, and brain connectivity using leave-one-out cross-validation.

\section{Materials and methods \\ Participants}

Seventy-four non-treatment-seeking adult smokers participated in the previously described study. ${ }^{6}$ Eight participants were excluded due to excessive ( $>1$ voxel) head motion during the MRI scan, leaving 66 participants ( 32 female; 5 left-handed) who passed MRI quality control for the final analysis. Participants reported their racial-ethnic characteristics as follows: 38 White, 22 African American, 4 Asian, and 2 Hispanic. Their ages ranged from 18 to 49 years (mean $\pm \mathrm{SE}=30.58 \pm 1.19$ ), with $14.13 \pm 0.23$ years of education. All 
participants gave written informed consent to participate in the protocol approved by the University of Pennsylvania Institutional Review Board. This study was conducted in accordance with the Declaration of Helsinki. Exclusion criteria were 1) presence of DSM-IV-TR Axis 1 psychiatric disorder, ${ }^{40}$ except for nicotine/tobacco use disorder; 2) urine drug screen positive for illicit opioids, benzodiazepines, cannabinoids, cocaine, or methamphetamine; 3) baseline urinary cotinine levels $<50 \mathrm{ng} / \mathrm{mL}^{41}$ 4) presence of medical or neurological disorder or treatment that may affect the cerebrovascular system; and 5) safety-related contraindications for MRI scanning.

\section{Materials and design}

One hundred ninety-nine 30-s long smoking-cessation videos targeting adult smokers were obtained from the collection of the Annenberg School of Communication. ${ }^{4-6,21}$

For each video, visual and auditory stimulation were quantified using the $\mathrm{I}^{2}$ instrument. ${ }^{20,21}$ For the visual $\mathrm{I}^{2}$, two trained coders viewed each video and located where camera changes occurred or visual structural features (eg, a video graphic) were introduced in a way that mimicked camera changes. For each camera change/structural feature, coders considered the amount of new visual information according to seven visual characteristics: change in emotionally arousing images, novel focal point of the scene, unrelatedness to the previous scene, new object, closer distance, different perspective, and form change. A camera change/structural feature could have a score ranging from 0 to 7 . For each video, the scores were summed across all camera changes/structural features, resulting in a total visual $\mathrm{I}^{2}$ score for that video. Similarly, for the auditory $\mathrm{I}^{2}$, two trained coders located where auditory structural features (eg, voice change, music onset) were introduced in each video. For each structural feature, the coders considered the presence of five auditory characteristics: new sound or silence, unrelatedness to the previous scene, voice change, emotional sound, and emotion change. The total score across all auditory structural features resulted in the auditory $\mathrm{I}^{2}$ for each video. Prior research showed high inter-coder reliability on the visual $\mathrm{I}^{2}$ (Krippendorff's $\alpha=0.94$ ) and auditory $\mathrm{I}^{2}$ (Kendall's $\tau=0.76$ ) scores. ${ }^{21}$

AS determination followed the procedure previously reported and utilized by previous studies. ${ }^{4-6}$ Briefly, two trained coders viewed the videos, transcribed the verbal arguments, and extracted the main arguments of each video. These arguments were then reviewed by two different coders who edited the text to capture all the content of the videos stated explicitly via voice and text on screen and implicitly through the visuals. The arguments extracted from each video were assessed for perceived AS in a survey of 387 current smokers, who were asked to rate the arguments from eight to twelve videos using an 11-item questionnaire (eg, "the statement is a reason for quitting smoking that is convincing"; 5-point scale, $1=$ strongly disagree, $5=$ strongly agree). A balanced design was used so that each video was rated by an average of 38 smokers. The means of the 38 raters for each argument were converted to $\mathrm{z}$ scores, and then those scores were summed across arguments for each video to produce an overall $\mathrm{z}$ score for each video. ${ }^{4-6}$

From the available 199 videos, we selected 32 videos whose AS scores fell in the top or bottom quartiles, yielding 16 high-AS and 16 low-AS videos. ${ }^{6}$ The high-AS and low-AS videos did not differ in visual $\mathrm{I}^{2}(38.88 \pm 5.40$ vs $30.25 \pm 5.84, \mathrm{t}(30)=-1.08, p=0.29)$ or auditory $\mathrm{I}^{2}(7.69$ \pm 1.09 vs $8.56 \pm 1.19, \mathrm{t}(30)=0.54, p=0.59)$. Participants were randomly assigned to either the high-AS condition $(\mathrm{N}=34)$ or the low-AS condition $(\mathrm{N}=32)$.

\section{fMRI task}

Participants underwent fMRI while watching either the 16 high-AS smoking-cessation videos or the 16 low-AS ones. For each participant, the videos were presented in a random order. Each video was preceded and followed by a 16-second rest period (grey crosshair on a homogenous black background). Each video was 30-s long and was presented only once.

\section{Behavioral assessments}

Sensation seeking was assessed using the Brief Sensation Seeking Scale (BSSS). ${ }^{42}$ It is an eight-item, self-report measure, in which respondents rate their agreement to each item on a 4-point scale (1=disagree strongly, $4=$ agree strongly). Higher scores indicate higher levels of sensation seeking. Participants' severity of nicotine dependence was assessed by the Fagerstrom Test of Nicotine Dependence (FTND). ${ }^{43}$ This is a six-item, self-report measure with a range of $0-10$, with higher scores reflecting greater nicotine dependence. Both BSSS and FTND have shown good test-retest reliability. ${ }^{42,44}$ Urine cotinine levels were measured using the HPLC tandem mass spectrometry system (Agilent Technologies) with a limit of detection of $2 \mathrm{ng} / \mathrm{mL}$. 6,41

\section{Procedure}

The study was comprised of phone screening, baseline assessment, a video+fMRI session, and a follow-up session 
1 month later. At the baseline session, participants were evaluated for eligibility, demographics, and handedness. ${ }^{45}$ One hour before the fMRI session, participants provided urine samples for baseline cotinine levels, reported the average number of cigarettes smoked per day in the past 30 days (CPD), and completed the BSSS and FTND. After assessments and 30-45 mins before the beginning of the fMRI session, participants were given an opportunity to smoke one of their own cigarettes outdoors under observation. All participants took the opportunity to smoke. Before the video task began, participants were instructed to attend to the videos. At the follow-up session approximately 30 days later (mean $\pm \mathrm{SE}=33.06 \pm 1.81 \mathrm{~d}$ ), the $\mathrm{CPD}$ and FTND scores were recorded, the video engagement test was completed, and the repeat urine samples for cotinine levels were obtained. Of the 66 participants who completed the fMRI session, all completed the baseline FTND and CPD, 65 completed the BSSS, 62 completed the baseline assessment of urine cotinine, 50 completed the follow-up CPD, 56 completed the follow-up FTND, and 51 completed the follow-up assessment of urine cotinine.

\section{fMRI data acquisition and processing}

MRI imaging was performed using Siemens Tim Trio 3T (Erlangen) system and a 32-channel receive-only head coil. Blood oxygenation level-dependent (BOLD) fMRI was performed, using a whole-brain, single-shot gradient-echo echo-planar sequence with the following parameters: $\quad \mathrm{TR} / \mathrm{TE}=2000 / 30 \mathrm{~ms}, \quad \mathrm{FOV}=220 \mathrm{~mm}$, matrix $=64 \times 64$, slice thickness $/$ gap $=3.4 / 0 \mathrm{~mm}, 32$ slices, effective voxel resolution of $3.4 \times 3.4 \times 3.4 \mathrm{~mm}^{3}$. fMRI is a non-invasive technique that has been widely used to investigate brain function. By measuring changes in the blood oxygenation level in the brain, BOLD fMRI allows detection of regional hemodynamic responses as a result of neural activities. ${ }^{46}$ After BOLD fMRI, 5 mins MPRAGE T1-weighted images were acquired as highresolution structural/anatomical images with the following parameters: $\mathrm{TR} / \mathrm{TE}=1630 / 3.87 \mathrm{~ms}, \mathrm{FOV}=250 \mathrm{~mm}$, matrix $=256 \times 192$, effective voxel resolution of $1 \times 1 \times 1$ $\mathrm{mm}^{3}$. An oblique acquisition, oriented along the anterior commissure-posterior commissure line allowed coverage of the entire brain with the exception of the lower cerebellum.

Imaging data were analyzed using SPM 12 (Wellcome Trust Centre for Neuroimaging, London, UK) following the guidelines by Friston et al (2007). ${ }^{47}$ The functional images were adjusted for slice timing and realigned to the first scan to correct for head motion. The structural images were coregistered to the functional images so that they are geometrically aligned to each other in the same coordinate system. The structural images were normalized into stereotactic Montreal Neurological Institutespace with 3-mm cubic voxels, and the resulting normalization parameters were then used to normalize the functional images. Lastly, the normalized functional images were spatially smoothed by a Gaussian filter with full-width/half-maximum parameter set to $8 \mathrm{~mm}$. Subject-level statistical analyses were performed voxelwise by modeling the videos using a canonical hemodynamic response function. Furthermore, the model included the normalized visual and auditory $\mathrm{I}^{2}$ scores in order to test the parametric modulation of visual information and auditory information. There was no correlation between visual and auditory $\mathrm{I}^{2}$ scores $(\mathrm{r}=-0.10$, $p=0.58$ ), indicating that the analysis did not suffer from multicollinearity. Six rigid body motion parameters were included as covariates, and the rest periods were treated as an implicit baseline. Significant activation at the grouplevel was identified at $p<0.05$ corrected for familywise error and cluster size $\mathrm{k}>50$ contingent voxels. One-sample t-tests across all participants were used to identify the visual and auditory cortices that responded to visual and auditory information by the contrast of visual vs auditory parametric modulation and vice versa. Independent-sample t-tests examined the differences between high-AS and lowAS participants in the neural response at the visual and auditory cortices.

Regions of interest (ROIs) were defined as spheres centered at the peak voxels of activation from the onesample t-tests with a radius of $5 \mathrm{~mm}$ using MarsBaR (http://marsbar.sourceforge.net). Specifically, the visual and auditory cortices were defined based on the parametric effects of visual and auditory $\mathrm{I}^{2}$ scores, respectively. The time series of the BOLD signal at the ROIs were extracted and high-pass filtered, and the effect of head motion was removed by regressing out the six motion parameters from the signal. The connectivity between the visual and auditory cortices was calculated as the Fisher-transformed correlation coefficient between the time series of the corresponding ROIs.

To test the specificity of the connectivity between visual and auditory cortices, we also examined the connectivity of a number of control ROIs defined by term-based metaanalysis in the Neurosynth database (neurosynth.org). These control ROIs (and their associated terms and coordinates) include the dorsal anterior cingulate cortex 
(term="attention", $\mathrm{x} / \mathrm{y} / \mathrm{z}=6 / 16 / 42$ ), bilateral dorsolateral prefrontal cortex (term="attention", $\mathrm{x} / \mathrm{y} / \mathrm{z}=-28 / 0 / 52 \&$ \& 28/-2/ $54)$, bilateral inferior parietal lobule (term="attention", $\mathrm{x} / \mathrm{y} /$ $z=-26 /-50 / 52 \& 26 /-56 / 52)$, ventromedial prefrontal cortex (term="default mode", $\mathrm{x} / \mathrm{y} / \mathrm{z}=-2 / 50 /-6$ ), precuneus (term="default mode", $\mathrm{x} / \mathrm{y} / \mathrm{z}=2 /-54 / 26$ ), bilateral angular gyrus (term="default mode", $x / y / z=-46 /-70 / 32 \& 50 /-62 /$ 32 ), bilateral amygdala (term="emotion", $x / y / z=-20 /-4 /-16$ $\& 24 /-4 /-16$ ), bilateral postcentral gyrus (term="somatosensory", $\mathrm{x} / \mathrm{y} / \mathrm{z}=-56 /-20 / 20$ \& 56/-18/38), bilateral insula (term="somatosensory", $\mathrm{x} / \mathrm{y} / \mathrm{z}=-36 /-12 / 8 \quad \& \quad 42 /-16 / 14$ ), paracentral lobule (term="somatosensory", $x / y / z=-10 /-34 /$ 60 ), mid-cingulate cortex (term="somatosensory", $\mathrm{x} / \mathrm{y} / \mathrm{z}=$ $-2 / 4 / 38$ ), supplementary motor area (term="somatosensory", $\mathrm{x} / \mathrm{y} / \mathrm{z}=-2 /-8 / 54$ ), bilateral thalamus (term="somatosensory", $\quad \mathrm{x} / \mathrm{y} / \mathrm{z}=-12 /-16 / 4 \quad \& \quad 14 /-18 / 10)$, bilateral hippocampus (term="episodic memory", $\mathrm{x} / \mathrm{y} / \mathrm{z}=-26 /-16 /-$ $20 \& 32 /-14 /-16$ ), and bilateral parahippocampal gyrus (ter$\mathrm{m}=$ "episodic memory", $\mathrm{x} / \mathrm{y} / \mathrm{z}=-22 /-30 /-14 \& 22 /-24 /-20)$.

\section{Moderated mediation analysis}

Moderated mediation analysis tests the extent to which the moderator influences the effect of the independent variable on the outcome variable through the mediator. ${ }^{36-38} \mathrm{We}$ conducted this analysis using a bootstrapping procedure implemented in the PROCESS macro for SPSS 22 (http:// www.processmacro.org). ${ }^{38,39}$ We tested the role of visual and auditory cortical connectivity as a mediator between AS condition (high vs low) and follow-up cotinine levels (adjusted for baseline levels), moderated by participants' levels of sensation seeking. Before analysis, follow-up cotinine levels were adjusted for the baseline levels. High-AS and low-AS groups were coded as 1 and -1 , respectively. The index of moderated mediation was computed to test for the significance of moderated mediation effects based on a bias-corrected bootstrap approximation with 5000 iterations. To visualize the moderating effect of sensation seeking, the association between brain connectivity and follow-up cotinine levels was plotted separately for smokers in the higher quartile of sensation seeking and those in the lower quartile. We also tested the significance of the direct effect of AS on cotinine levels that did not go through the mediator, in order to determine whether brain connectivity fully or partially mediated AS's effect on cotinine levels. Lastly, we examined the contribution of brain connectivity to the prediction of follow-up cotinine levels by comparing the moderated mediation model to a reduced model with only $\mathrm{AS}$ and sensation seeking as predictors.

\section{Prediction analysis}

We used the leave-one-out cross-validation analysis to test the predictive power of the moderated mediation model on follow-up cotinine levels (adjusted for baseline). We performed a regression analysis on all but one participant, with cotinine levels as the dependent variable and with independent variables of AS, visual-auditory cortical connectivity, sensation seeking, and the interaction between connectivity and sensation seeking. The parameter estimates obtained from the regression model were then applied to the omitted participant, yielding a predicted value of his/her cotinine level. After repeating this procedure in every participant, the inaccuracy of prediction was estimated by the mean squared error (MSE), that is, MSE= $\left[\sum_{i}\left(\hat{Y}_{-i}-Y_{i}\right)^{2}\right] / N$, where $N$ is the number of participants, $Y_{i}$ is the actual cotinine level for the $i$-th participant, and $\hat{Y}_{-i}$ is the predicted cotinine level for the $\mathrm{i}$-th participant based on the regression model with all but the i-th participant. The significance of MSE was determined by a permutation test. ${ }^{48}$ For each of the 5,000 permutations, a random MSE (ie, $\left.\mathrm{MSE}_{\text {null }}\right)$ was calculated after permuting the cotinine levels while leaving the independent variables fixed. This yielded an empirical distribution of prediction inaccuracy under the null hypothesis that the cotinine levels could not be predicted by AS, visual-auditory cortical connectivity, sensation seeking, or the interaction between connectivity and sensation seeking. The significance of the original MSE was then calculated as $\operatorname{Prob}\left(\mathrm{MSE} \geq \mathrm{MSE}_{\text {null }}\right)$.

\section{Results}

\section{Demographic and behavioral variables}

Participants' baseline and follow-up demographic and behavioral measures are summarized in Table 1. The high-AS and low-AS groups did not differ on any of these measures ( $p s>0.17$ ), except that the high-AS group's follow-up cotinine levels were significantly lower than the low-AS group after controlling for baseline cotinine levels $(\mathrm{F}(1,46)=6.01, p<0.02)$.

\section{fMRI results}

The amount of visual information in the smoking-cessation videos, as indicated by the visual $\mathrm{I}^{2}$ scores, was positively associated with the neural response in the occipital cortex $(\mathrm{k}=5539, \mathrm{Z}=8.33, \mathrm{x} / \mathrm{y} / \mathrm{z}=27 /-67 /-10)$ (Figure 1, regions in red-yellow). The amount of auditory information, as 
Table I Summary of behavioral measures ( $\mathrm{N}$ or mean $\pm \mathrm{SE})$

\begin{tabular}{|l|l|l|}
\hline & High-AS group & Low-AS group \\
\hline Baseline & & \\
Gender & 18 male, I6 female & 16 male, 16 female \\
Race & 21 Cau, 8 AA, 3 Asian, 2 Hisp & 17 Cau, I4 AA, I Asian \\
Age (years) & $29.68 \pm 1.54$ & $31.53 \pm 1.83$ \\
Education (years) & $14.09 \pm 0.31$ & $14.17 \pm 0.35$ \\
BSSS & $2.89 \pm 0.10$ & $2.84 \pm 0.11$ \\
FTND & $4.38 \pm 0.40$ & $4.56 \pm 0.43$ \\
Cigarettes per day & $14.59 \pm 1.23$ & $15.06 \pm 1.28$ \\
Cotinine (ng/mL) & $1451.47 \pm 388.81$ & $1524.70 \pm 225.20$ \\
\hline Follow-up & & \\
FTND & $4.23 \pm 0.45$ & $4.23 \pm 0.54$ \\
Cigarettes per day & $13.93 \pm 1.19$ & $15.00 \pm 1.74$ \\
Cotinine (ng/mL) & $1142.80 \pm 205.02$ & $1770.95 \pm 297.88$ \\
\hline
\end{tabular}

Abbreviations: AS, argument strength; BSSS, Brief Sensation Seeking Scale; FTND, Fagerstrom Test of Nicotine Dependence; Cau, Caucasian; AA, African American; Hisp, Hispanic.

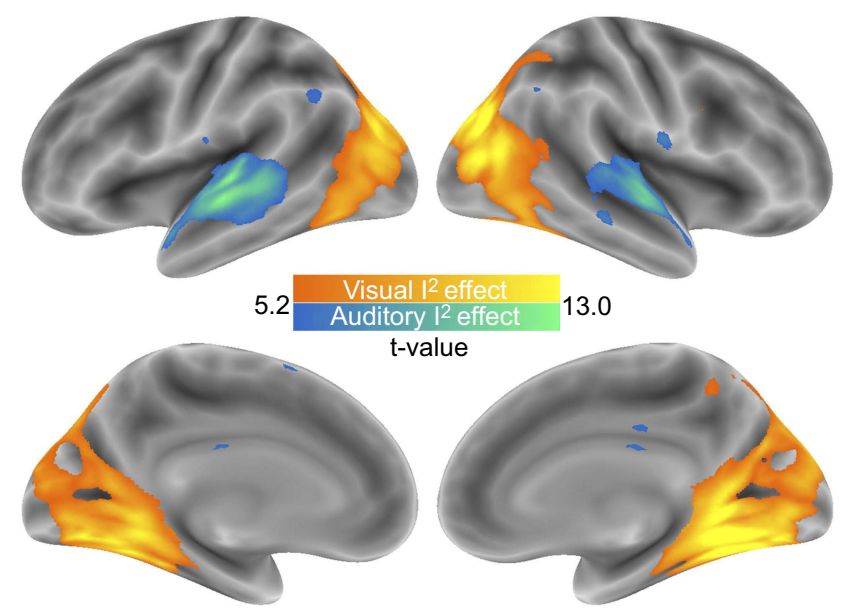

Figure I Brain responses associated with the visual and auditory $\mathrm{I}^{2}$ scores $(p<0.05$ corrected for familywise error).

indicated by the auditory $\mathrm{I}^{2}$ scores, was positively associated with neural response in the bilateral posterior superior temporal cortex (left: $\mathrm{k}=909, \mathrm{Z}=7.82, \mathrm{x} / \mathrm{y} / \mathrm{z}=-57 /-22 /-1$; right: $k=808, Z=7.46, \mathrm{x} / \mathrm{y} / \mathrm{z}=60 /-10 /-1)$ as well as the right parietal cortex $(k=56, Z=6.04, \mathrm{x} / \mathrm{y} / \mathrm{z}=51 /-61 / 47)$ and the thalamus $(\mathrm{k}=78, \mathrm{Z}=5.82, \mathrm{x} / \mathrm{y} / \mathrm{z}=-6 /-4 / 8)$ (Figure 1 , regions in bluegreen). The activity in these regions did not differ between high-AS and low-AS groups. There was a positive connectivity between the visual and auditory cortices (mean $\pm \mathrm{SE}=0.62 \pm 0.03, \mathrm{t}(65)=23.60, p<0.0001)$, which was significantly greater in the high-AS group compared to the low-AS group $(0.73 \pm 0.03$ vs $0.50 \pm 0.03, \mathrm{t}(64)=5.13, p<0.00001)$. The connectivity among the control ROIs as well as their connectivity with the visual and auditory cortices did not differ between the high-AS and low-AS groups $(\mathrm{t}(64)=-1.94-1.59$, $p \mathrm{~s}>0.05$ ).

\section{Moderated mediation analysis}

To test our hypotheses, we ran a mediated moderation model such that visual-auditory cortical connectivity mediated the effect of AS on the follow-up cotinine levels (adjusted for baseline), and that the mediation was further moderated by sensation seeking (Figure 2, upper panel). Table 2 shows that sensation seeking significantly moderated the indirect effect of AS on the follow-up cotinine levels through brain connectivity (index of moderated mediation $=40.52, \mathrm{SE}=18.45$, $p=0.01$ ). Lower sensation seeking was associated with greater mediation effect. The moderated mediation effect was not significant for the connectivity among the control ROIs as well as their connectivity with the visual and auditory cortices ( $p s>0.05)$.

Estimations of individual paths are shown in Table 2. Consistent with our first hypothesis, high-AS smoking-cessation videos produced greater connectivity between the visual and auditory cortices than the low-AS videos (Figure 2, lower left panel). Consistent with our second hypothesis, the association between brain connectivity and follow-up cotinine levels (adjusted for baseline) was moderated by sensation seeking, such that greater connectivity predicted less smoking only among low-sensation-seeking smokers (Figure 2, lower right panel). We confirmed our previous report ${ }^{6}$ by showing a significant total effect of AS on cotinine, but the direct effect was not significant, suggesting a full mediation effect. 


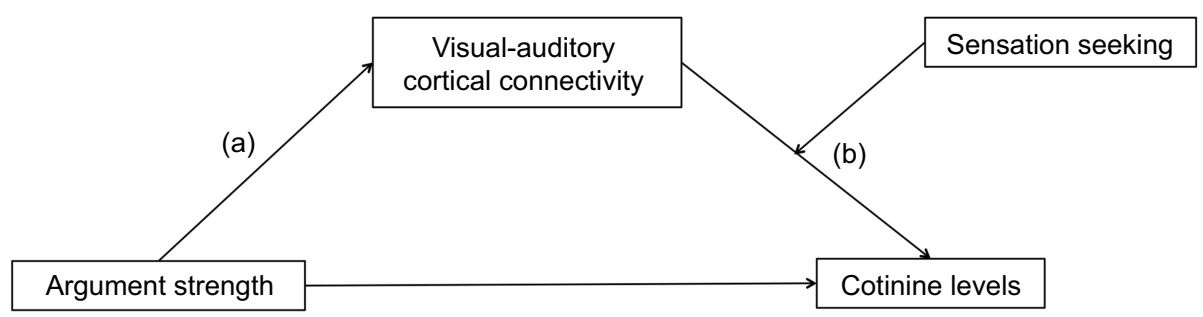

(a)

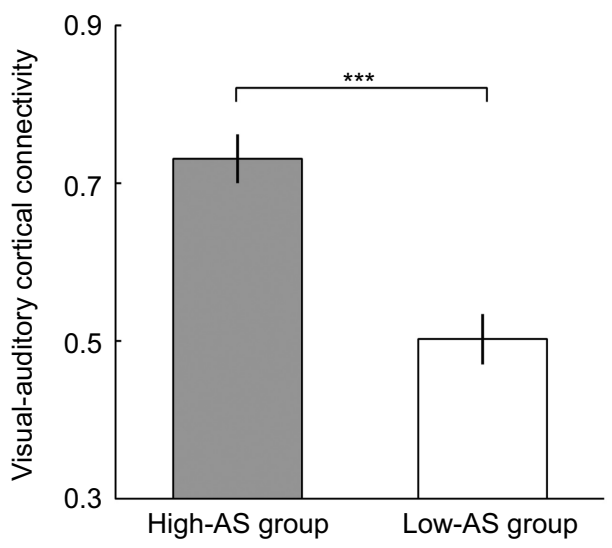

(b)

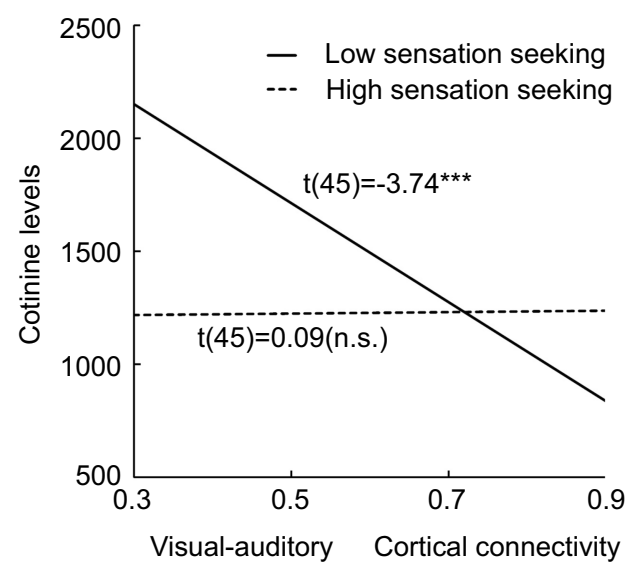

Figure 2 Upper panel: the moderated mediation model with sensation seeking moderating the indirect effect of argument strength on cotinine levels through visual-auditory cortical connectivity. Lower left panel: smokers who watched the high-argument strength (AS) videos showed greater connectivity between the visual and auditory cortices than those who watched the low-AS ones. Lower right panel: greater visual-auditory cortical connectivity ( $x$-axis) was associated with reduced cotinine levels ( $y$-axis) among smokers in the lower quartile but not higher quartile of sensation seeking.

Compared to the model with only AS and sensation seeking as predictors, the addition of brain connectivity and its interaction with sensation seeking led to a significantly improved model fit $\left(\Delta \mathrm{R}^{2}=0.18, \mathrm{~F}(2,44)=7.29\right.$, $p=0.002$ ).

\section{Prediction analysis}

Leave-one-out cross-validation ( $p<0.0002$; Figure 3 ) confirmed the predictive power of the above moderated mediation model - that is, the follow-up cotinine levels could be predicted sufficiently well by the moderated mediation model.

\section{Discussion}

We showed that high-AS smoking-cessation videos induced greater connectivity between the occipital and bilateral posterior temporal cortices. These regions are involved in the encoding of visual and auditory information, respectively. The findings extend our previous study ${ }^{6}$ which showed that videos with higher AS are more effective in changing smokers' subsequent smoking behavior. The connectivity between the visual and auditory cortices in turn showed a negative association with future smoking behavior in smokers low in sensation seeking. Such association was not found for the connectivity of brain regions underlying attention, default mode, emotion, somatosensory processing, or episodic memory. These findings provide experimental support to the hypotheses that multisensory brain connectivity mediates the behavioral effectiveness of high-AS smoking-cessation videos, but that the strength of the mediation depends on the individual's level of sensation seeking.

Our study is the first to show that the visual and auditory components of the $\mathrm{I}^{2}$ measure are linked to the neural activity and the visual and auditory cortices, respectively, during viewing of videos. The visual and auditory cortices are connected both anatomically ${ }^{49}$ and functionally. ${ }^{23}$ The activity in the auditory cortex is modulated by visual input, ${ }^{50}$ and the visual cortical activity can in turn be modulated by auditory input. ${ }^{24}$ Such neural connectivity has been shown to underlie concurrent processing of visual and auditory information. ${ }^{15-17,51}$ The connectivity is further modulated by factors such as the synchrony between the visual and auditory input ${ }^{16,17}$ and one's familiarity with the multimodal stimuli. ${ }^{52}$ Our findings extend prior knowledge by showing that smoking-cessation videos with higher AS induced greater occipitotemporal connectivity, 
Table 2 Results of the moderated mediation model

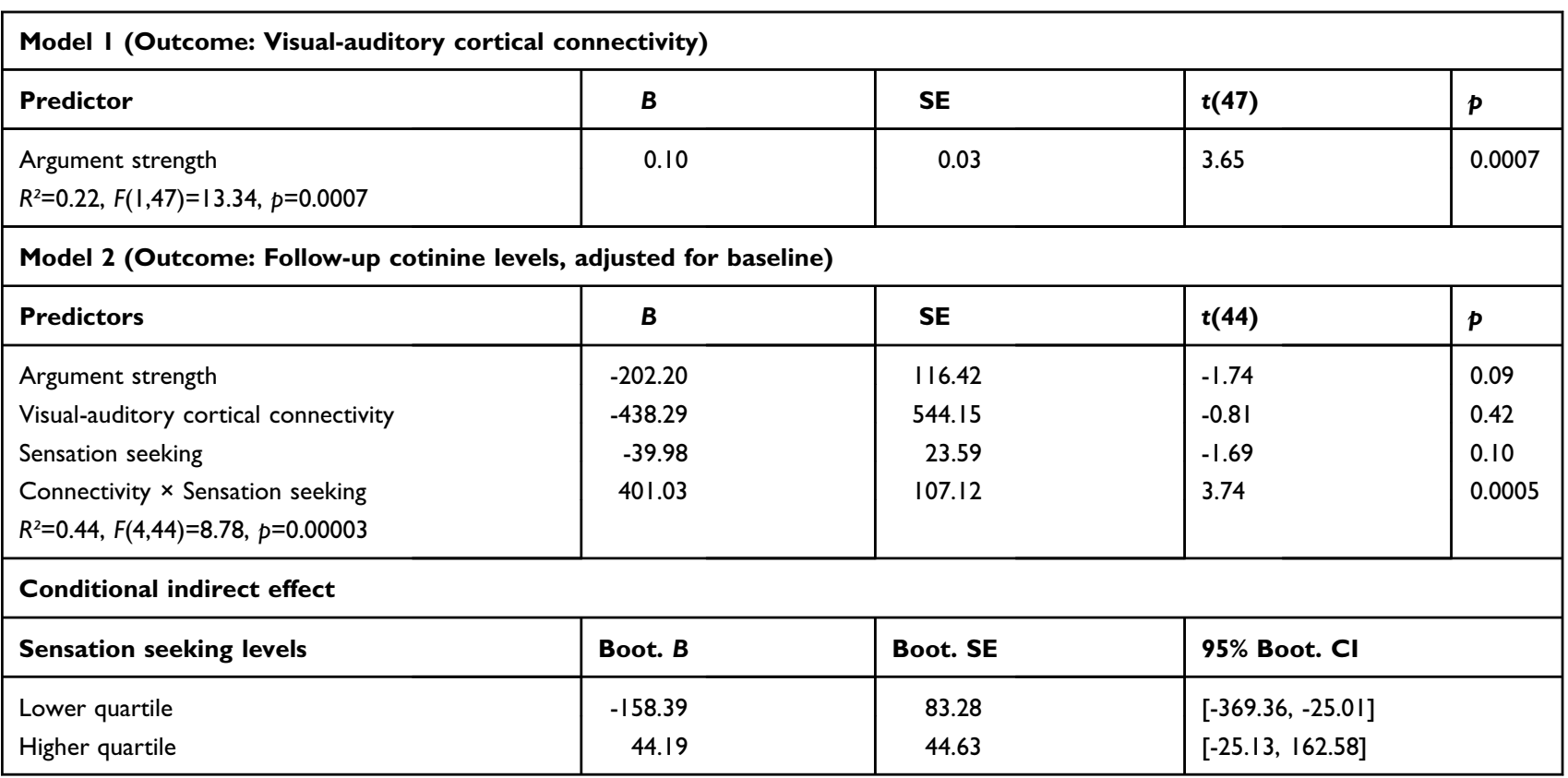

Abbreviation: Boot., bootstrapping with 5,000 iterations.

suggesting that such connectivity is a marker of the persuasiveness of complex sensory stimuli.

Results of prior studies of the link between sensory processing and outcomes of public health video messages have been inconsistent. For example, neural activity at the sensory cortices in response to safe-sex videos was negatively correlated with change in attitudes toward condom use, ${ }^{13}$ while the association between activity in these regions in response to smoking-cessation videos and reduction in smoking was positive. ${ }^{53}$ One potential source of this apparent discrepancy may be variability in the ads' AS. ${ }^{6}$ The present study showed that connectivity mediated the effect of AS on the low-sensation-seeking smokers' subsequent smoking behavior. This suggests that the connectivity between the visual and auditory cortices may better reflect the effectiveness of video messages than the independent measures of visual and auditory cortical activity. Integration of multimodal information may be an intermediate mechanism underlying effective processing of persuasive video messages.

The role sensation seeking plays in various substance use disorders including smoking is well established. ${ }^{30,31,54,55}$ However, data on the relationship between sensation seeking and reducing or quitting smoking is inconclusive - some studies show that high-sensation-seeking smokers are less compliant with cessation treatment ${ }^{56}$ and less likely to quit smoking, ${ }^{57,58}$ whereas other studies have found no association between sensation seeking and readiness to change smoking behavior $^{59}$ or quit attempts. ${ }^{60}$ Our findings suggest an alternative explanation for this inconsistency by showing that although both high- and low-sensation seeking smokers may respond to smoking-cessation videos, low-sensation-seeking smokers are more readily influenced by the integration of visual and auditory information in high-AS videos. Smokers higher in sensation seeking appeared to exhibit a trend for lower levels of cotinine at follow-up, regardless of visualauditory integration. According to the moderated mediation model, the inability of high-sensation seeking smokers to benefit from high-AS videos is unlikely to be due to poor processing of the videos, given similar multisensory processing between high and low sensation seekers. Thus, they may have responded to other characteristics of the videos, with brain activity that was not related to the connectivity between visual and auditory cortices. We believe that an important next step is to explore how anti-smoking communication (as well as other public health messaging) helps high sensation seekers improve their control over reward-pursuing behaviors.

Our findings may have implications on health behavior management from two perspectives. First, they point to greater integration of visual and auditory information as an independent measure of effectiveness of a video. Such measure could be used to evaluate future public health video ads. Future design of health promotion video ads should not only focus on the level of visual and auditory stimulation, but also the coherence of the two modalities. Second, we proposed a moderated mediation model that could predict future health 


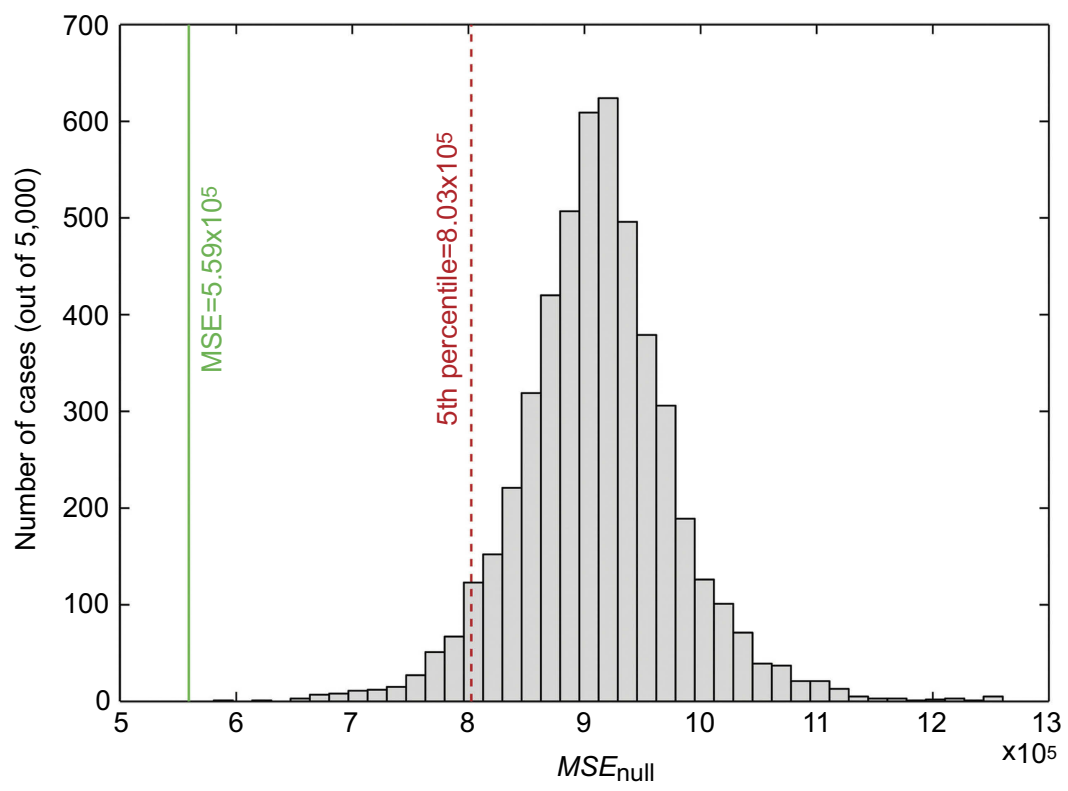

Figure 3 Leave-one-out cross-validation showing that the moderated mediation model was predictive of follow-up cotinine levels with significantly smaller prediction error (ie, MSE) as compared to the null distribution of prediction error ( $\left.\mathrm{MSE}_{\text {null }}\right)$ obtained through 5,000 permutations. Green solid line: actual MSE value produced by the original data. Red dashed line: 5 th percentile of the empirical $M_{S E} E_{\text {null }}$ produced by permutation.

Abbreviation: MSE, mean squared error of prediction.

behavior using baseline variables including sensation seeking, brain connectivity, and AS of videos. Specifically, the addition of brain connectivity to the model significantly improved the prediction. Although fMRI is not practical in some research sttings, our findings can be applied to techniques that are more accessible, especially the electroencephalogram and the functional near-infrared spectroscopy, which could be used to assess brain connectivity. Successful prediction of future behavior change will allow early identification of individuals that are less likely to benefit from health communications and may thus require additional interventions.

The present study comes with a number of limitations. First, we did not test whether the association between AS and audiovisual integration is specific to smokers and smoking-cessation videos. Although the potential implications for health communications do not depend on such specificity, it would be interesting for future studies to investigate whether our findings extend to health promotion videos on other public health issues. Second, although we showed that watching high-AS videos induced greater visual-auditory cortical connectivity, it remains to be determined whether such connectivity is associated with greater effectiveness of video stimuli. It is possible that increasing the connectivity could improve the comprehension of the messages. It would also be important to examine whether viewers with greater connectivity will find the videos more persuasive. Third, it is unclear how long the moderated mediation effect would last beyond the 1-month followup. Future longitudinal research could address this question by having a longer follow-up and by testing the outcome of repeated exposure to the messages. Fourth, research with adolescents suggests that it is the overlap between measured sensation seeking and impulsivity, but not the attraction to exciting sensation, that underlies the persistence of smoking and use of other drugs. ${ }^{61,62}$ Future studies should examine to what extent the effects of sensation seeking are attributable to impulsivity. Fifth, we observed that the visual $\mathrm{I}^{2}$ scores were positively associated with the neural activity of a substantial area of the visual cortex that likely spanned the extrastriate visual cortical areas (including V2, V3, V4, and V5), but not the primary visual cortex V1. This is consistent with the nature of visual $\mathrm{I}^{2}$ which mainly indexes the amount of complex visual information such as camera change, new objects and novel focal point of the scene, instead of low-level properties of visual stimulation such as brightness and smoothness. It is possible different subregions of the visual cortex were engaged in different aspects of visual processing during our task. However, given the complexity of our video stimuli, we were not able to achieve a fine-grained mapping of visual processing onto the brain. We hope that future research will address this issue by using more advanced analytical techniques. Lastly, 
although we only focused on the sensory cortices, many other brain regions are also involved in the processing of the smoking-cessation videos. ${ }^{6}$ One of the most important regions is the medial prefrontal cortex, whose response to health messages has been shown to predict future health behavior change. ${ }^{6,10,11}$ Likewise, besides sensation seeking, responsiveness to smoking-cessation interventions may also depend on other factors ranging from biological, such as nicotine metabolic rate, ${ }^{63,64}$ to socio-psychological, such as susceptibility to peer influence. ${ }^{65,66}$ A replication in a larger sample, and with a wider range of biological and behavioral variables, could provide a better understanding of how different factors, including the activity and connectivity of other brain regions, interact to mediate and influence the efficacy of anti-smoking as well as other types of health messaging.

\section{Conclusions}

Our findings highlight the importance of multisensory integration in the processing of smoking-cessation videos by showing that the connectivity between visual and auditory cortices mediated the greater efficacy of high- vs lowAS videos for smokers with lower levels of sensation seeking. These findings may help to inform future efforts to refine health communication strategies so that smokers with different levels of sensation seeking and related traits will be more able to reduce and eventually quit smoking.

\section{Acknowledgments}

The study was supported by the Annenberg Public Policy Center of the University of Pennsylvania and by grants from the National Institutes of Health (DA036028, DA028874, and HD084746).

\section{Disclosure}

The authors report no conflicts of interest in this work.

\section{References}

1. Pierce JP, Anderson DM, Romano RM, Meissner HI, Odenkirchen JC. Promoting smoking cessation in the United States: effect of public service announcements on the Cancer Information Service telephone line. J Natl Cancer Inst. 1992;84(9):677-683. doi:10.1093/jnci/84.9.677

2. Elder RW, Shults RA, Sleet DA, Nichols JL, Thompson RS, Rajab W. Effectiveness of mass media campaigns for reducing drinking and driving and alcohol-involved crashes: a systematic review. $\mathrm{Am} J$ Prev Med. 2004;27(1):57-65. doi:10.1016/j.amepre.2004.03.002

3. Siska M, Jason J, Murdoch P, Yang WS, Donovan RJ. Recall of AIDS public service announcements and their impact on the ranking of AIDS as a national problem. Am J Public Health. 1992;82(7):1029-1032. doi:10.2105/ajph.82.7.1029
4. Strasser AA, Cappella JN, Jepson C, et al. Experimental evaluation of antitobacco PSAs: effects of message content and format on physiological and behavioral outcomes. Nicotine Tob Res. 2009;11(3):293302. doi: $10.1093 / \mathrm{ntr} / \mathrm{ntn} 026$

5. Zhao X, Strasser A, Cappella J, Lerman C, Fishbein M. A measure of perceived argument strength: reliability and validity in health communication contexts. Commun Methods Meas. 2011;5(1):48-75. doi: $10.1080 / 19312458.2010 .547822$

6. Wang A-L, Ruparel K, Loughead JW, et al. Content matters: neuroimaging investigation of brain and behavioral impact of televised antitobacco public service announcements. J Neurosci. 2013;33 (17):7420-7427. doi:10.1523/JNEUROSCI.3840-12.2013

7. Johnson BT, Eagly AH. Effects of involvement on persuasion: a meta-analysis. Psychol Bull. 1989;106(2):290-314. doi:10.1037/ 0033-2909.106.2.290

8. Wood W, Quinn JM. Forewarned and forearmed? Two meta-analytic syntheses of forewarnings of influence appeals. Psychol Bull. 2003;129(1):119-138.

9. Ramsay IS, Yzer MC, Luciana M, Vohs KD, MacDonald AW III. Affective and executive network processing associated with persuasive antidrug messages. J Cogn Neurosci. 2013;25(7):1136-1147. doi:10.1162/jocn_a_00391

10. Falk EB, Berkman ET, Mann T, Harrison B, Lieberman MD. Predicting persuasion-induced behavior change from the brain. J Neurosci. 2010;30 (25):8421-8424. doi:10.1523/JNEUROSCI.0063-10.2010

11. Falk EB, Berkman ET, Whalen D, Lieberman MD. Neural activity during health messaging predicts reductions in smoking above and beyond self-report. Health Psychol. 2011;30(2):177-185. doi:10.1037/a0022259

12. Langleben DD, Loughead JW, Ruparel K, et al. Reduced prefrontal and temporal processing and recall of high "sensation value" ads. NeuroImage. 2009;46(1):219-225. doi:10.1016/j.neuroimage.2008.12.062

13. Seelig D, Wang A-L, Jaganathan K, et al. Low message sensation health promotion videos are better remembered and activate areas of the brain associated with memory encoding. PLoS One. 2014;9(11): e113256. doi:10.1371/journal.pone. 0113256

14. Petty R, Cacioppo J. Communication and Persuasion: Central and Peripheral Routes to Attitude Change. New York, NY: Springer Verlag; 1986.

15. Senkowski D, Schneider TR, Foxe JJ, Engel AK. Crossmodal binding through neural coherence: implications for multisensory processing. Trends Neurosci. 2008;31(8):401-409. doi:10.1016/j.tins.2008.05.002

16. Lewis R, Noppeney U. Audiovisual synchrony improves motion discrimination via enhanced connectivity between early visual and auditory areas. $J$ Neurosci. 2010;30(37):12329-12339. doi:10.1523/ JNEUROSCI.5745-09.2010

17. Werner S, Noppeney U. Distinct functional contributions of primary sensory and association areas to audiovisual integration in object categorization. J Neurosci. 2010;30(7):2662-2675. doi:10.1523/ JNEUROSCI.5091-09.2010

18. Molholm S, Ritter W, Javitt DC, Foxe JJ. Multisensory visual-auditory object recognition in humans: a high-density electrical mapping study. Cereb Cortex. 2004;14(4):452-465. doi:10.1093/cercor/bhh007

19. Morgan SE, Palmgreen P, Stephenson MT, Hoyle RH, Lorch EP. Associations between message features and subjective evaluations of the sensation value of antidrug public service announcements. J Commun. 2003;53(3):512-526. doi:10.1111/j.1460-2466.2003. tb02605.x

20. Lang A, Bradley SD, Park B, Shin M, Chung Y. Parsing the resource pie: using STRTs to measure attention to mediated messages. Media Psychol. 2006;8:369-394. doi:10.1207/s1532785xmep0804_3

21. Lee S, Cappella JN, Lerman C, Strasser AA. Smoking cues, argument strength, and perceived effectiveness of antismoking PSAs. Nicotine Tob Res. 2011;13(4):282-290. doi:10.1093/ntr/ntq255

22. Friston KJ. Functional and effective connectivity: a review. Brain Connect. 2011;1(1):13-36. doi:10.1089/brain.2011.0008 
23. Eckert MA, Kamdar NV, Chang CE, Beckmann CF, Greicius MD, Menon V. A cross-modal system linking primary auditory and visual cortices: evidence from intrinsic fMRI connectivity analysis. Hum Brain Mapp. 2008;29(7):848-857. doi:10.1002/hbm.20560

24. Zangenehpour S, Zatorre RJ. Crossmodal recruitment of primary visual cortex following brief exposure to bimodal audiovisual stimuli. Neuropsychologia. neuropsychologia.2009.10.022

25. Hur YM, Bouchard TJ Jr. The genetic correlation between impulsivity and sensation seeking traits. Behav Genet. 1997;27(5):455-463.

26. Quinn PD, Harden KP. Differential changes in impulsivity and sensation seeking and the escalation of substance use from adolescence to early adulthood. Dev Psychopathol. 2013;25(1):223-239. doi:10.1017/ S0954579412000284

27. Zuckerman M. Sensation Seeking: Beyond the Optimal Level of Arousal. Hillsdale, NJ: Lawrence Erlbaum; 1979.

28. Zuckerman M. Sensation Seeking and Risky Behavior. Washington, DC: American Psychological Association; 2007.

29. Carton S, Jouvent R, Widlocher D. Sensation seeking, nicotine dependence, and smoking motivation in female and male smokers. Addict Behav. 1994;19(3):219-227.

30. Crawford AM, Pentz MA, Chou CP, Li CY, Dwyer JH. Parallel developmental trajectories of sensation seeking and regular substance use in adolescents. Psychol Addict Behav. 2003;17(3):179-192. doi:10.1037/0893-164X.17.3.179

31. Zuckerman M, Neeb M. Demographic influences in sensation seeking and expressions of sensation seeking in religion, smoking and driving habits. Pers Individ Dif. 1980;1(3):197-206. doi:10.1016/0191-8869 (80)90051-3

32. Benowitz NL, Hukkanen J, Jacob P III. Nicotine chemistry, metabolism, kinetics and biomarkers. Handb Exp Pharmacol. 2009; (192):29-60.

33. Pokorski TL, Chen WW, Bertholf RL. Use of urine cotinine to validate smoking self-reports in U.S. Navy recruits. Addict Behav. 1994;19(4):451-454.

34. Wall MA, Johnson J, Jacob P, Benowitz NL. Cotinine in the serum, saliva, and urine of nonsmokers, passive smokers, and active smokers. Am J Public Health. 1988;78(6):699-701. doi:10.2105/ajph.78.6.699

35. Zhu AZ, Renner CC, Hatsukami DK, et al. The ability of plasma cotinine to predict nicotine and carcinogen exposure is altered by differences in CYP2A6: the influence of genetics, race, and sex. Cancer Epidemiol Biomarkers Prev. 2013;22(4):708-718. doi:10.1158/1055-9965.EPI-12-1234-T

36. Edwards JR, Lambert LS. Methods for integrating moderation and mediation: a general analytical framework using moderated path analysis. Psychol Methods. 2007;12(1):1-22. doi:10.1037/1082989X.12.1.1

37. Preacher KJ, Rucker DD, Hayes AF. Addressing moderated mediation hypotheses: theory, methods, and prescriptions. Multivar Behav Res. 2007;42(1):185-227. doi:10.1080/00273170701341316

38. Hayes AF. An index and test of linear moderated mediation. Multivar Behav Res. 2015;50(1):1-22. doi:10.1080/00273171.2014.962683

39. Preacher KJ, Hayes AF. Asymptotic and resampling strategies for assessing and comparing indirect effects in multiple mediator models. Behav Res Methods. 2008;40(3):879-891.

40. American Psychiatric Association. Diagnostic and Statistical Manual of Mental Disorders, Text Revision (DSM-IV-TR). Washington, DC: American Psychiatric Association; 2000.

41. SRNT. Subcommittee on Biochemical Verification. Biochemical verification of tobacco use and cessation. Nicotine Tob Res. 2002;4 (2):149-159. doi:10.1080/14622200210123581

42. Hoyle RH, Stephenson MT, Palmgreen P, Lorch EP, Donohew RL. Reliability and validity of a brief measure of sensation seeking. Pers Individ Dif. 2002;32(3):401-414. doi:10.1016/S0191-8869(01) 00032-0
43. Heatherton TF, Kozlowski LT, Frecker RC, Fagerstrom KO. The Fagerstrom test for nicotine dependence: a revision of the Fagerstrom Tolerance Questionnaire. Br J Addict. 1991;86(9):11191127.

44. Pomerleau CS, Carton SM, Lutzke ML, Flessland KA, Pomerleau OF. Reliability of the Fagerstrom tolerance questionnaire and the Fagerstrom test for nicotine dependence. Addict Behav. 1994;19 (1):33-39.

45. Oldfield RC. The assessment and analysis of handedness: the Edinburgh inventory. Neuropsychologia. 1971;9(1):97-113.

46. Heeger DJ, Ress D. What does fMRI tell us about neuronal activity? Nat Rev Neurosci. 2002;3(2):142-151. doi:10.1038/nrn730

47. Friston KJ, Penny WD, Ashburner J, Kiebel SJ, Nichols TE. Statistical Parametric Mapping: The Analysis of Functional Brain Images. London, UK: Elsevier Science; 2007.

48. Anderson MJ. Permutation tests for univariate or multivariate analysis of variance and regression. Can J Fish Aquat Sci. 2001;58 (3):626-639. doi:10.1139/f01-004

49. Beer AL, Plank T, Greenlee MW. Diffusion tensor imaging shows white matter tracts between human auditory and visual cortex. Exp Brain Res. 2011;213(2-3):299-308. doi:10.1007/ s00221-011-2715-y

50. Besle J, Fischer C, Bidet-Caulet A, Lecaignard F, Bertrand O, Giard MH. Visual activation and audiovisual interactions in the auditory cortex during speech perception: intracranial recordings in humans. $J$ Neurosci. 2008;28(52):14301-14310. doi:10.1523/JNEUROSCI.28 75-08.2008

51. Noppeney U, Ostwald D, Werner S. Perceptual decisions formed by accumulation of audiovisual evidence in prefrontal cortex. $J$ Neurosci. 2010;30(21):7434-7446. doi:10.1523/JNEUROSCI.045510.2010

52. Schall S, von Kriegstein K. Functional connectivity between facemovement and speech-intelligibility areas during auditory-only speech perception. PLoS One. 2014;9(1):e86325. doi:10.1371/journal.pone. 0086325

53. Shi Z, Wang AL, Fairchild VP, Romer D, Langleben DD Individual differences in the processing of anti-smoking public service announcements: an imaging genetics study. The 22nd Annual Meeting of the Society for Research on Nicotine \& Tobacco; 2016; Chicago, IL.

54. Newcomb MD, Mcgee L. Influence of sensation seeking on general deviance and specific problem behaviors from adolescence to young adulthood. J Pers Soc Psychol. 1991;61(4):614-628.

55. Pedersen W. Mental health, sensation seeking and drug use patterns: a longitudinal study. Br J Addict. 1991;86(2):195-204.

56. Kahler CW, Spillane NS, Metrik J, Leventhal AM, Monti PM. Sensation seeking as a predictor of treatment compliance and smoking cessation treatment outcomes in heavy social drinkers. Pharmacol Biochem Behav. 2009;93(3):285-290.

57. Lipkus IM, Barefoot JC, Williams RB, Siegler IC. Personality measures as predictors of smoking initiation and cessation in the UNC Alumni Heart Study. Health Psychol. 1994;13(2):149-155.

58. Brook JS, Zhang C, Balka EB, Seltzer N, Brook DW. Personality characteristics in the mid-forties predict women's smoking cessation in their mid-sixties. Psychol Rep. 2013;113(3):921-929. doi:10.2466/ 09.10.13.PR0.113x26z9

59. Harmsen H, Bischof G, Brooks A, Hohagen F, Rumpf HJ. The relationship between impaired decision-making, sensation seeking and readiness to change in cigarette smokers. Addict Behav. 2006;31(4):581-592. doi:10.1016/j.addbeh.2005.05.038

60. Hall PA, Fong GT, Yong HH, Sansone G, Borland R, Siahpush M. Do time perspective and sensation-seeking predict quitting activity among smokers? Findings from the International Tobacco Control (ITC) Four Country Survey. Addict Behav. 2012;37(12):1307-1313. doi:10.1016/j.addbeh.2012.06.022 
61. Khurana A, Romer D, Betancourt LM, Hurt H. Working memory ability and early drug use progression as predictors of adolescent substance use disorders. Addiction. 2017;112(7):1220-1228. doi:10.1111/add.13792

62. Khurana A, Romer D, Betancourt LM, Hurt H. Modeling trajectories of sensation seeking and impulsivity dimensions from early to late adolescence: universal trends or distinct sub-groups?. J Youth Adolesc. 2018;47 (9): 1992-2005.

63. Chen LS, Bloom AJ, Baker TB, et al. Pharmacotherapy effects on smoking cessation vary with nicotine metabolism gene (CYP2A6). Addiction. 2014;109(1):128-137. doi:10.1111/add.12353

64. Schnoll RA, Patterson F, Wileyto EP, Tyndale RF, Benowitz N, Lerman C. Nicotine metabolic rate predicts successful smoking cessation with transdermal nicotine: a validation study. Pharmacol Biochem Behav. 2009;92(1):6-11. doi:10.1016/j.pbb.2008.10.016
65. Paek H, Gunther A. How peer proximity moderates indirect media influence on adolescent smoking. Commun Res. 2007;34:407-432. doi:10.1177/0093650207302785

66. Gunther AC, Bolt D, Borzekowski DLG, Liebhart JL, Dillard JP. Presumed influence on peer norms: how mass media indirectly affect adolescent smoking. J Commun. 2006;56(1):52-68. doi:10.1111/ j.1460-2466.2006.00002.x

\section{Publish your work in this journal}

Psychology Research and Behavior Management is an international, peer-reviewed, open access journal focusing on the science of psychology and its application in behavior management to develop improved outcomes in the clinical, educational, sports and business arenas. Specific topics covered in the journal include: Neuroscience, memory and decision making; Behavior modification and management; Clinical applications; Business and sports performance management; Social and developmental studies; Animal studies. The manuscript management system is completely online and includes a very quick and fair peer-review system, which is all easy to use. Visit http://www. dovepress.com/testimonials.php to read real quotes from published authors. 